cover layer on a silicon substrate. While normal atomic force microscopy (AFM) showed a smooth, featureless surface of the top polymeric layer, the SNFUH phase image clearly showed the dispersed, buried gold nanoparticles.

SNFUH is particularly well suited for detecting defects and voids in microelectronics structures. For example, the scale of fabrication continues to shrink and interconnect metal lines are approaching 60-nm widths. Model shallow trench structures were fabricated in a dielectric material with a 50-nm-thick silicon nitride layer deposited as a capping layer and etched into the trenches. A 500-nm-thick lowdielectric polymer layer was deposited by spin-coating and annealing. A conventional topography scan showed a uniform and continuous polymer coating at the bottoms of the trenches as well as on the tops of the lines, as seen in Figure 1 (p. 169). However, the corresponding SNFUH phase image revealed embedded voids within the polymer and at the $\mathrm{SiN}$-polymer interfaces.

The SNFUH technique can also be used to image embedded or buried sub- structures in biological samples. A nearcontact mode was used for imaging soft structures, wherein the probe tip is lifted by $2-5 \mathrm{~nm}$ after it touches the surface. This was used to obtain high-contrast, high-resolution images of malaria parasites inside infected red blood cells. The direct real-space in vitro imaging was performed without labeling or sectioning the cells under physiologically viable conditions. SNFUH was thus demonstrated to be a versatile technique for nondestructive, high-resolution, real-space imaging of diverse materials systems. In particular, it fills the spatial resolution gap at the 10-100-nm scale for nondestructive subsurface imaging.

GOPAL RAO

\section{Silica Nanoparticles Doped with Multiple Fluorescent Dyes Demonstrated as Potential New Barcoding Tags}

Often in biological imaging experiments, it is desirable to view several components of a structure simultaneously in real time. This can be done with small-molecule fluo- rescent tags, but this technique is limited by the number of available dyes that are excited at the same wavelength but have distinguishable emission spectra. Quantum dots, polymer microspheres, and other materials have been used to overcome this limitation and create "bio-barcoding" systems, but many of these have problems with performance. In the January 11 issue of Nano Letters (p. 84; DOI: 10.1021/ n1052105b), researchers L. Wang and $W$. Tan from the University of Florida at Gainesville have presented a novel solution to this problem. The researchers incorporated combinations of three common fluorophores into silica nanoparticles (NPs) to create NPs that respond to monochromatic illumination with a range of distinguishable emission signatures. They also demonstrated the potential for using these materials as tags for biological molecules by functionalizing the NPs with biotin and binding them to avidinfunctionalized microspheres.

The researchers incorporated the organic dyes FITC, R6G, and ROX into silica NPs in a variety of ratios. Alone, these dyes

\section{www.mrs.org}

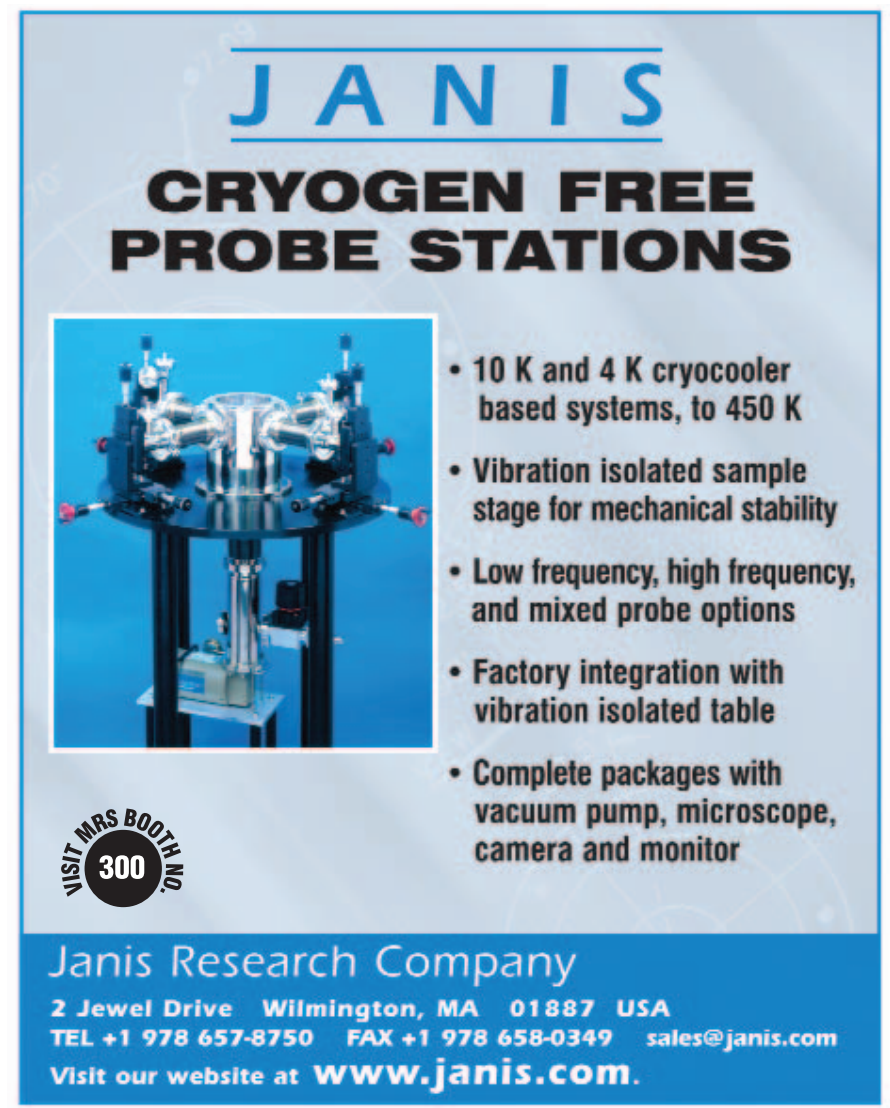

For more information, see http://www.mrs.org/bulletin_ads

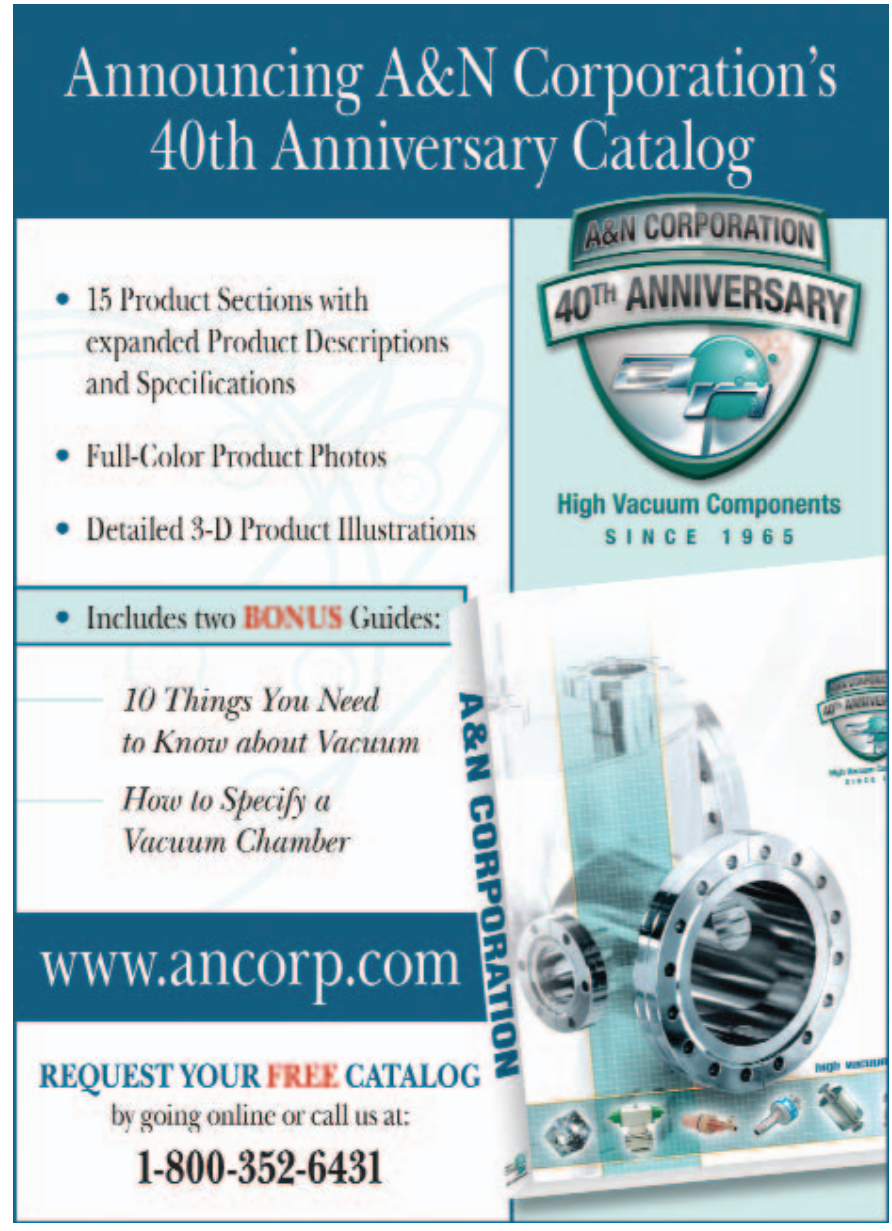

For more information, see http://www.mrs.org/bulletin_ads 
have different excitation maxima, but when incorporated together they form a tandem system for fluorescent resonant energy transfer (FRET) so that when excited at the optimal wavelength for FITC, the dye-impregnated silica NPs emit unique colors based on their dye ratios. The researchers synthesized the NPs by a modified Stöber synthesis route, in which the dyes were incorporated into the structure as the NPs grew. The NPs thus prepared were found to be uniform in size, colloidally stable, and strongly fluorescent.

To demonstrate the utility of the material, the researchers derivatized aminemodified NPs with biotin and attached them to avidin-derivatized microspheres. Under confocal fluorescent microscopy, these microsphere-NP complexes emitted light at the characteristic wavelength of the particular NP they contained. The researchers said that this approach can be generalized to label the NPs with other biopolymers for use in barcoding assays and multiplex bioanalysis. According to the researchers, the NPs can also be used as optical materials for display technologies.

KRISTA L. NIECE

Highly Ordered $\mathrm{TiO}_{2}$ Nanotube Array Improves Dye-Sensitized Solar Cells

Dye-sensitized solar cells (DSCs) are potentially a cheap and environmentally friendly alternative to silicon-based photovoltaics. Based on an effect analogous to photosynthesis, DSCs have achieved lightto-electricity conversion efficiencies of more than $11 \%$. In the February 8 issue of Nano Letters (p. 215; DOI: 10.1021/ nl052099j), G.K. Mor, C.A. Grimes, and colleagues at the Pennsylvania State University have reported the fabrication of a DSC based on an ordered array of $\mathrm{TiO}_{2}$ nanotubes. Although the photocurrent efficiency of their device is only about $3 \%$, their results suggest that nanotube-based DSCs may achieve conversion efficiencies 10 times as high, significantly exceeding silicon-based solar cells.

Standard DSCs are typically based on a $10-\mu m$-thick film of randomly arranged $\mathrm{TiO}_{2}$ nanoparticles. The nanoparticles are coated with a molecular layer of an organic dye (often extracted from blackberries, raspberries, or pomegranates) that releases electrons when illuminated with sunlight. The large surface area of the dye-coated nanoparticles enhances the efficiency of the light collection, and the released electrons become the device's photocurrent. However, the mobility of the released electrons is limited by scattering at the disordered boundaries between nanoparticles, which reduces the overall efficiency. To address this problem, the Penn State team grew ordered arrays of $\mathrm{TiO}_{2}$ nanotubes $360 \mathrm{~nm}$ in length on glass substrates by anodizing a 500-nm-thick titanium film, and then immersed the arrays in a ruthenium-based dye. When exposed to full sunlight, the resulting devices generated photocurrent with a relatively low efficiency of $2.9 \%$. However, the lifetime of liberated electrons was significantly longer than in nanoparticle devices, implying much less electron scattering by the ordered nanotube array.
The team concluded that the conversion efficiency was limited by the relatively short length of the nanotubes, and that by improving the fabrication procedure to grow micrometer-scale nanotubes, the efficiency could be boosted to close to the ideal limit of $31 \%$. If this proves to be the case, high-efficiency, dye-sensitized solar cells could become an important competitor to standard silicon solar cells, both in terms of lower

\section{Cost-Effective Portable Spin Coater}

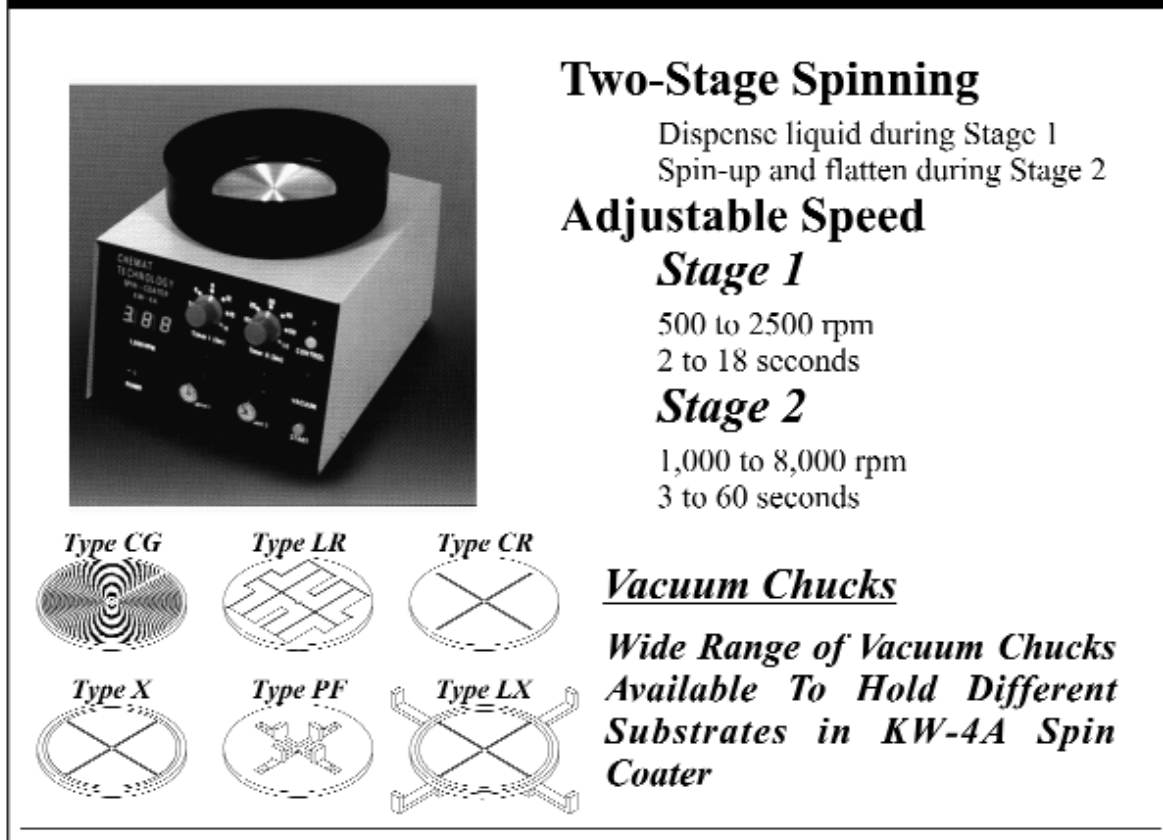

KW-4A SERIES PRODUCT LINE

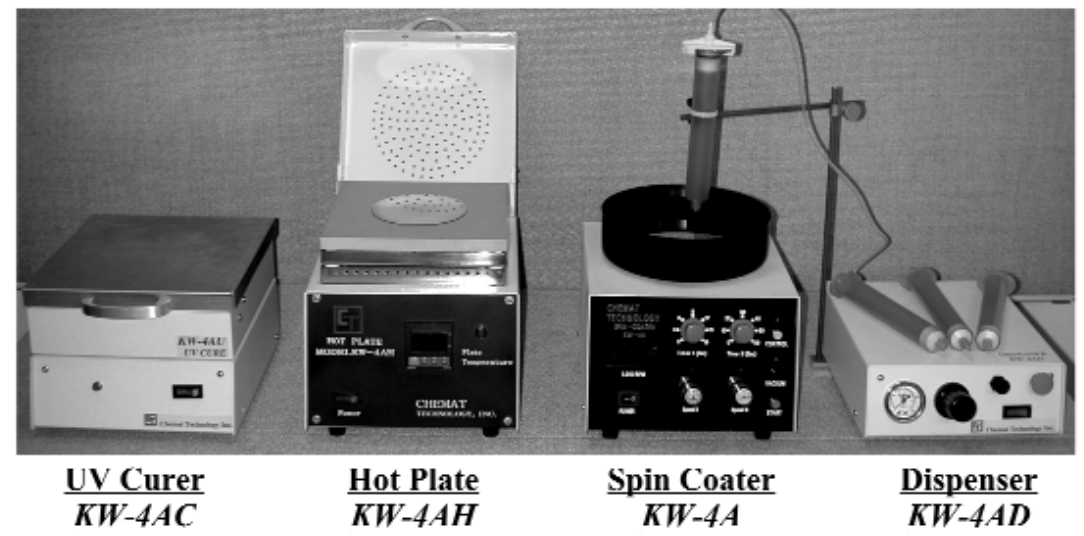

CHEMAT TECHNOLOGY, INC. 9036 Winnetka Avenue, Northridge, C $\wedge \mathbf{~} 91324$ 1-800-475-3628, Fax: 818-727-9477 